\title{
Family history and the natural history of colorectal cancer: systematic review
}

\author{
Nora B. Henrikson, PhD, MPH${ }^{1}$, Elizabeth M. Webber, $\mathrm{MS}^{2}$, Katrina A. Goddard, $\mathrm{PhD}^{2}$, \\ Aaron Scrol, MA ${ }^{1}$, Margaret Piper, PhD, MPH², Marc S. Williams, MD³, Doris T. Zallen, PhD, \\ Ned Calonge, MD, MPH${ }^{5}$, Theodore G. Ganiats, MD ${ }^{6}$, A. Cecile J.W. Janssens, MSc, PhD"7, \\ Ann Zauber, $\mathrm{PhD}^{8}$, Iris Lansdorp-Vogelaar, $\mathrm{PhD}^{9}$, Marjolein van Ballegooijen, $\mathrm{MD}, \mathrm{PhD}^{9}$ \\ and Evelyn P. Whitlock, MD, $\mathrm{MPH}^{2}$
}

\begin{abstract}
Purpose: Family history of colorectal cancer (CRC) is a known risk factor for CRC and encompasses both genetic and shared environmental risks.
\end{abstract}

Methods: We conducted a systematic review to estimate the impact of family history on the natural history of CRC and adherence to screening.

Results: We found high heterogeneity in family-history definitions, the most common definition being one or more first-degree relatives. The prevalence of family history may be lower than the commonly cited $10 \%$, and confirms evidence for increasing levels of risk associated with increasing family-history burden. There is evidence for higher prevalence of adenomas and of multiple adenomas in people with family history of CRC but no evidence for differential adenoma location or adenoma progression by family history. Limited data regarding the natural history of CRC by family history suggest a differential age or stage at cancer diagnosis and mixed evidence with respect to tumor location. Adherence to recommended colonoscopy screening was higher in people with a family history of CRC.

Conclusion: Stratification based on polygenic and/or multifactorial risk assessment may mature to the point of displacing family historybased approaches, but for the foreseeable future, family history may remain a valuable clinical tool for identifying individuals at increased risk for CRC.

Genet Med advance online publication 15 January 2015

Key Words: colorectal cancer; family history; risk stratification; systematic review
Colorectal cancer (CRC) ranks third in cancer incidence and death in the United States. Approximately $4 \%$ of CRC cases occur in those younger than 50 years of age. ${ }^{1}$ Hereditary conditions such as familial adenomatous polyposis and Lynch syndrome confer an extremely high lifetime risk of CRC but account for a minority of all CRC cases. A much larger proportion of US adults have moderately elevated risk of CRC due to a family history of CRC, probably reflecting a combination of shared polygenic and shared environmental risk..$^{2-4}$

Early detection of CRC through screening with established modalities beginning at age 50 reduces CRC morbidity and mortality, but adherence to CRC screening remains below the Centers for Disease Control and Prevention's goal of $80 \% .5,6$ Optimal screening for people with established family history of CRC is not as well defined, and screening recommendations vary, focusing on earlier initiation of screening, frequency of screening, or early screening in people in racial/ethnic groups. ${ }^{7-12}$ If optimal screening strategies could be determined based on evidence-based risk, and if adherence to screening could be improved, then there would be significant potential for further public health impact. Statistical modeling can give valuable information regarding how different screening practices might impact population outcomes, but populationbased, high-quality epidemiologic data are needed to inform such models.

We conducted a systematic review to identify evidence for the impact of family history of CRC on the risk and natural history of CRC and on screening adherence. The review was commissioned by the Evaluation of Genomic Applications in Practice and Prevention Working Group and was designed to inform a decision-analytic model of optimal screening strategies conducted by the National Cancer Institute Cancer Intervention and Surveillance Modeling Network consortium microsimulation modeling group. ${ }^{13}$ Because of the lack of studies at the time of the review suggesting clinical utility of polygenic models of moderate-penetrance gene variants for assessing CRC risk, we focused the review on the evidence for family history.

We systematically reviewed four areas, as follows. (i) Prevalence: what is the prevalence of a positive family history of CRC or adenoma in the population? Does prevalence vary

${ }^{1}$ Group Health Research Institute, Seattle, Washington, USA; ${ }^{2}$ Kaiser Permanente Center for Health Research, Portland, Oregon, USA; ${ }^{3}$ Geisinger Health System, Genomic Medicine Institute, Danville, Pennsylvania, USA; ${ }^{4}$ Virginia Tech, Blacksburg, Virginia, USA; ${ }^{5}$ The Colorado Trust, Denver, Colorado, USA; ${ }^{6}$ University of California, San Diego, San Diego, California, USA $;{ }^{7}$ Emory University, Atlanta, Georgia, USA $;{ }^{8}$ Memorial Sloan-Kettering Cancer Center, New York, New York, USA; ${ }^{9}$ Department of Health, Erasmus MC, Rotterdam, The Netherlands. Correspondence: Nora B. Henrikson (henrikson.n@ghc.org) 
by age, sex, or race/ethnicity of the person at risk for family history? (ii) Risk: what are the absolute and relative risks for CRC or adenoma associated with positive family history of CRC? (iii) Natural history: how does a positive family history of CRC affect the natural history of CRC and adenomas? (iv) Adherence: how does family history of CRC affect adherence to colonoscopy?

\section{MATERIALS AND METHODS}

\section{Search strategy and study selection}

Systematic literature searches were performed through 20 February 2013 in Medline, PubMed, and the Centre for Reviews and Dissemination. Searches were broadly scoped, using terms for CRC, family history, natural history, and screening adherence (Supplementary Material online). Two investigators independently reviewed identified abstracts and articles against a priori-specified inclusion criteria. Disagreements were resolved through consensus or input of a third reviewer. Details of search strategies, study selection, analysis, and results are available in the Supplementary Material.

Inclusion criteria for all questions included age of the person at risk $>18$ years and study reported in the English language. Exclusion criteria for all questions included CRC associated with illness (e.g., Crohn disease), inherited CRC syndromes, and studies with unmeasured or poorly defined family-history criteria. Only studies reporting family history in terms of both number and degree of relatedness for affected family members were included.

For risk, prevalence, and adherence, we included only population-based estimates. Case-control studies were excluded to minimize bias by enrichment with people with family history of CRC. For natural history we included only studies that would allow assessment by family history of age at onset of adenoma or CRC, number of adenomas or tumors, advanced adenoma, and adenoma/tumor location. Case-only and case-control studies were permitted for this question.

We reviewed all included studies for the independence of their study populations and years of data collection. For studies from the same data source, we included articles that best fit the relevance to the study questions.

Because of the large number of studies and the heterogeneity of outcomes and family-history definitions, we further refined our inclusion criteria using a best-evidence approach, a staged method for prioritizing evidence from all potentially relevant to a set best suited to answering a question. Such approaches can be appropriate for large, heterogeneous literature to enhance applicability of the evidence. ${ }^{14}$ For risk and prevalence, we defined our best-evidence set as population-based studies with a minimum sample size of 30,000-a number chosen by team consensus-and excluded screening studies because these may overrepresent people with family history. For natural history, we limited our scope to studies in which colonoscopy was conducted in a minimum sample size of 500 people with CRC or adenoma. For adherence, we identified an existing comprehensive review that contained data highly relevant to our study question ${ }^{15}$ and included this as a source of primary evidence, ${ }^{16}$ supplemented by subsequent US population-based studies.

\section{Data abstraction, synthesis, and quality assessment}

Histological characteristics of tumors and adenomas of interest for this review were informed by the World Health Organization histology resource ${ }^{17}$ in consultation with clinical experts (Supplementary Material online). We abstracted estimates of prevalence or relative risk, with confidence intervals (CIs) when provided. For the adherence question, we limited data abstraction to adults 50 years of age or older to reflect current screening recommendations. We did not conduct meta-analyses due to the heterogeneity of outcome measures and family-history definitions. We critically appraised the best-evidence set for threats to internal validity from selection, attrition, detection, and reporting bias, adapting criteria from previously published approaches. ${ }^{15,18}$

\section{RESULTS}

Based on review of 3,271 abstracts, 437 articles met the inclusion criteria. Of those, 224 were excluded after review of the full text for risk, prevalence, and natural history; 96 were excluded for adherence. After applying best-evidence criteria, our data set included 30 unique articles: 8 addressed prevalence, 9 addressed risk, 9 addressed natural history, and 11 addressed adherence (Figure 1).

\section{Prevalence}

Summary. The prevalence of having at least one first-degree relative (FDR) with CRC was estimated between 3.1 and $10 \%$. The prevalence of having at least two FDRs with CRC was much lower (0.3-0.34\%). Having an FDR with an early age at CRC diagnosis (before age 45 or 50 years) was uncommon, approximately $0.3 \%$, as compared with having an FDR with a later CRC diagnosis (older than age 60 or 65 years), which was more than $3 \%$. Few data were available regarding racial and ethnic-specific prevalence of family history.

Study details. Eight studies met our best-evidence criteria: ${ }^{2,19-25}$ six were from the United States, one was from the United Kingdom, ${ }^{23}$ and one was from Japan. ${ }^{21}$ All had approximately equal representation of males and females, except for two studies that included women only. ${ }^{24,25}$ The average age in the study populations was between 50 and 60 years, except for one ${ }^{20}$ which had an average age of 39.3 years. All studies but one $^{19}$ ascertained family-history information from unverified self-report (Table 1).

The most common definition of family history was having one or more FDRs (1+FDR) with a CRC diagnosis. Five studies estimated the prevalence of having 1+ FDR with a CRC diagnosis, with estimates ranging between 3.1 and $10 \%{ }^{2,19,23-25}$ The only study with objective measures of both family history and CRC diagnosis estimated a positive family-history prevalence of $4.1 \% .^{19}$ The lowest estimate was from a study that evaluated only mothers and daughters and likely underestimates the 


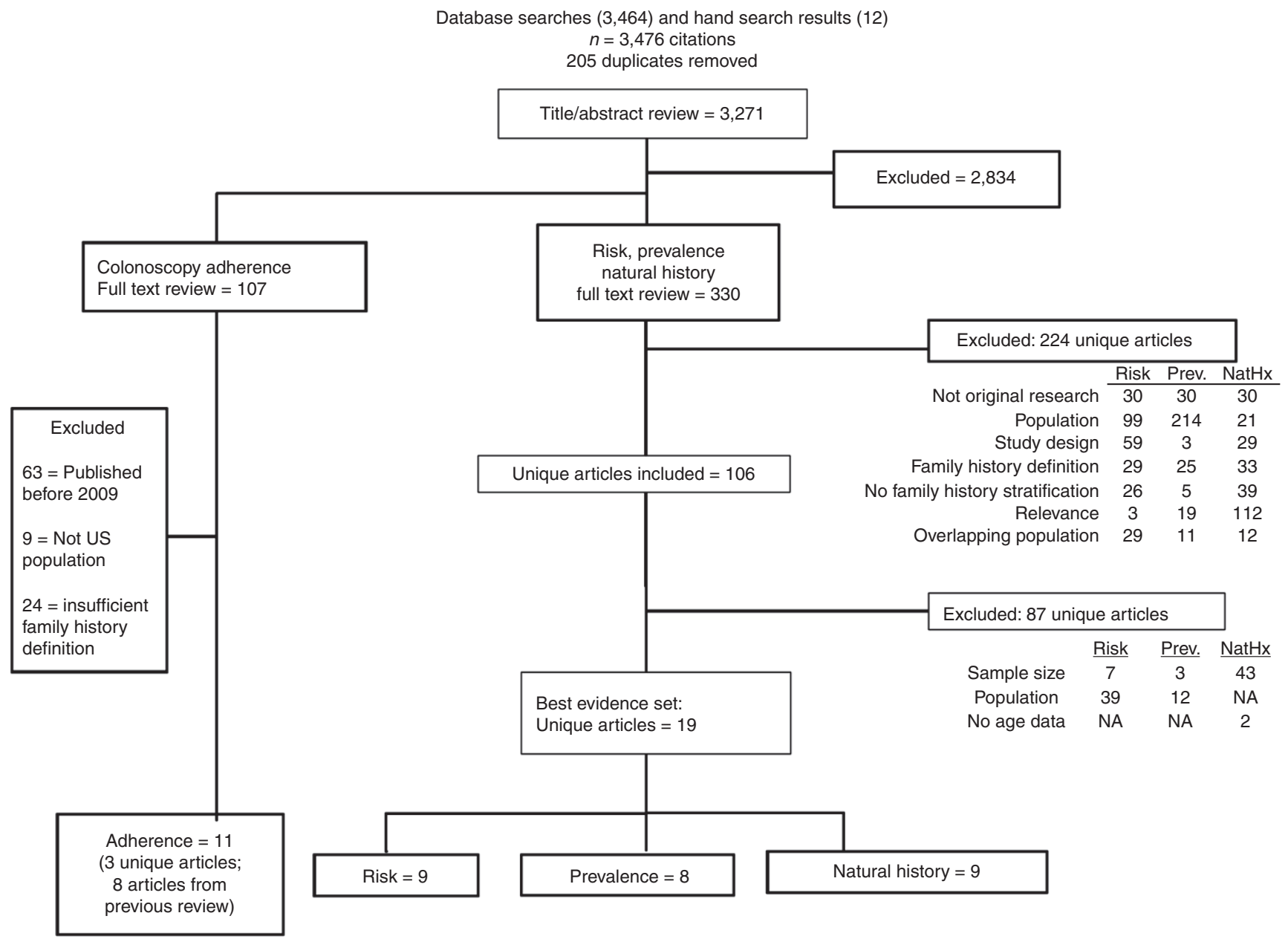

Figure 1 Article flow diagram.

prevalence of a positive family history. Two studies provided estimates for the prevalence of having exactly one affected FDR, which were 3.9 and $9.4 \%{ }^{19,22}$ One study provided an estimate for having at least one affected parent (1.7\%). ${ }^{21}$ The prevalence of having at least two affected FDRs was 0.3 to $0.34 \%{ }^{19,23}$

Scheuner et al. ${ }^{20}$ estimated the prevalence of multiple familyhistory configurations grouped together in risk groups. The "moderate risk" group-defined as either one FDR with lateonset cancer or two second-degree relatives (SDRs) from the same lineage with late-onset cancer or one SDR with earlyonset cancer and other SDRs with associated cancers-had a prevalence of $4.2 \%$. Two studies considered the relative's age at diagnosis as part of the definition of family history. ${ }^{19,23}$ As expected, having an FDR diagnosed at age younger than 45 (prevalence $0.36 \%{ }^{23}$ ) or 50 years (prevalence $0.27 \%{ }^{19}$ ) was much less common than having an FDR diagnosed at age older than $60(3.4 \%)^{19}$ or $65(4.1 \%)^{23}$ years.

Two studies considered the effect of demographic characteristics. Both found a higher prevalence of family history in females as compared with males $(6.1 \%$ of men and $7.4 \%$ of women with at least one affected $\mathrm{FDR}^{23}$ and $4.5 \%$ of men as compared with $6.2 \%$ of women in the author-defined "strong" or "moderate" risk groups). ${ }^{20}$ "Moderate" or "strong" family history was also higher among whites than among other racial or ethnic groups in the same study (7.3\% white, $2.5 \%$ Latino, $4.1 \%$ Asian, 6.1\% African American, 4.4\% other). ${ }^{20}$

\section{Risk}

Summary. The relative risk of developing CRC varied from 0.89 (for people with no family history) to nearly a 20 -fold risk (for people with likely inherited syndromes); risk levels in between had increasing family-history burden. Risk of CRC was higher when the relative was diagnosed at a younger age. CRC risk also depended on the age of the person at risk: people with positive family history in their 30s or 40s demonstrated a higher relative risk as compared with their age-matched peers than people with the same positive family history at an older age.

Nine studies of unique populations ranging in size from 30,353 to 7 million individuals were included., ${ }^{2,19-21,23-27}$ Five studies were conducted in the United States, ${ }^{2,19,20,24,25}$ and four others were conducted in Britain, ${ }^{23}$ Sweden, ${ }^{27}$ Japan, ${ }^{21}$ and China. ${ }^{26}$ Most studies considered the risk of developing CRC in those with family histories of CRC as compared with those with no family history. One very large study considered standardized 
Table 1 Prevalence of family history

\begin{tabular}{|c|c|c|c|c|c|c|}
\hline Author (year) & Data source & $N$ & $\begin{array}{l}\text { Mean age } \\
\text { (range) }\end{array}$ & $\%$ Female & Family-history categories & Prevalence (\%) \\
\hline \multirow{3}{*}{ Taylor $(2010)^{19}$} & \multirow{3}{*}{$\begin{array}{l}\text { Utah Population } \\
\text { Database }\end{array}$} & \multirow{3}{*}{$2,327,327$} & \multirow{3}{*}{$N R(N R)$} & \multirow{3}{*}{ NR } & $1+\mathrm{FDR}$ & 4.1 \\
\hline & & & & & 2 FDR & 0.31 \\
\hline & & & & & $2+F D R$ & 0.34 \\
\hline \multirow{2}{*}{ Scheuner $(2010)^{20}$} & \multirow{2}{*}{2005 CHIS } & \multirow{2}{*}{$33,187^{a}$} & \multirow{2}{*}{39.3} & \multirow{2}{*}{$\begin{array}{l}50 \% \\
\text { (Weighted) }\end{array}$} & $\begin{array}{l}\text { EITHER } 1 \text { FDR *or* } 2 \text { SDRs } d x \text { age }>50 * \text { or* } 1 \\
\text { SDR dx age }<50 \text { with CRC and } 1+\text { SDR dx age } \\
>50 \text { with endometrial cancer }\end{array}$ & 4.2 \\
\hline & & & & & $\begin{array}{l}\text { EITHER } 1+\text { FDR } d x \text { age }<50 * \text { or }^{*} 2+\text { ADR *or* } \\
\text { hereditary syndrome }\end{array}$ & 1.1 \\
\hline Pinsky $(2003)^{22}$ & PLCO & 149,332 & $N R(55-74)$ & $51 \%$ & $2+F D R$ & 0.7 \\
\hline \multirow[t]{4}{*}{ Sandhu $(2001)^{23}$} & \multirow[t]{4}{*}{ EPIC-Norfolk (UK) } & \multirow[t]{4}{*}{$30,353^{c}$} & \multirow[t]{4}{*}{59.1} & \multirow[t]{4}{*}{$55 \%$} & 1+ FDR with CRC & $6.8^{d}$ \\
\hline & & & & & $2+F D R$ & $0.3^{d}$ \\
\hline & & & & & EITHER 2 FDR *or* 1 FDR $d x$ age $<45$ & 0.6 \\
\hline & & & & & $1+$ FDR dx age $<45,45-64, \geq 65$ & $0.3,2.0,3.8^{d}$ \\
\hline Poole $(1999)^{24}$ & CPS-1 & 429,483 & 51 & $100 \%$ & 1+ FDR with CRC & 3.7 \\
\hline Nelson $(1993)^{25}$ & $\begin{array}{l}\text { lowa Women's } \\
\text { Health Study }\end{array}$ & 40,657 & $N R(55-69)$ & $100 \%$ & 1+ Mother/daughter with CC & 3.1 \\
\hline Fuchs $(1994)^{2}$ & NHS & 87,031 & 49.1 & $100 \%$ & 1+ FDR with CRC & $9.4^{\mathrm{e}}$ \\
\hline Fuchs $(1994)^{2}$ & HPFS & 32,085 & 51.5 & $0 \%$ & 1+ FDR with CRC & $10^{e}$ \\
\hline
\end{tabular}

ADR, any-degree relative; CC, colon cancer; CHIS, California Health Interview Survey; Cl, confidence interval; CPS, Cancer Prevention Study; CRC, colorectal cancer; dx, diagnosed; EPIC, European Prospective Investigation into Cancer; FDR, first-degree relative; HPFS, Health Professional Followup Study; JACC, Japan Collaborative Cohort Study for Evaluation of Cancer Risk; NHS, Nurses Health Study; NR, not reported; PLCO, prostate, lung, colorectal, and ovarian cancer screening trial; SDR, second-degree relative.

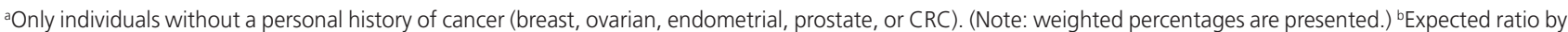

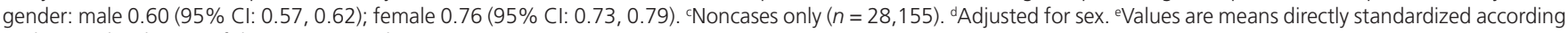
to the age distribution of the respective cohort in its entirety.

incidence ratios for those with CRC with family history as compared with the general population. ${ }^{19}$ Summarized results for risk are shown in Table 2; detailed tables are in the Supplementary Material online.

The Utah Population Database (UPDB) is a statewide population-based resource of genealogies of the original Utah pioneers and their modern-day descendants. This analysis used Utah Cancer Registry data (from 1952) linked to the UPDB in a subset of 2.3 million persons who were part of three generations of Utah genealogy data and descendants of original Utah pioneers.

Analyses from the UPDB using detailed family pedigrees found distributions of standardized incidence ratios for CRC ranging from 0.89 (95\% CI: $0.87-0.91$ ) for those with no FDR with CRC to 19.86 (95\% CI: 9.29-43.24) for those with five or more FDRs with CRC, who likely have inherited syndromes. ${ }^{19}$ Significant differences in CRC incidence were present between those with confirmed negative family history (0.89), one FDR with date of diagnosis unknown or after age 60 (1.91-1.99), one FDR diagnosed before age 60 (2.69), and three or four FDRs with CRC (4.41). The Nurses' Health Study found increased risk for CRC from 1+ FDR with CRC to 2+ FDRs with CRC. ${ }^{2}$ In the UPDB, having an FDR diagnosed with CRC before age 60 years increased personal CRC risk. Other US data were consistent, with an earlier diagnosis of CRC in an FDR conferring higher personal CRC risk, but age ranges were not consistently defined (i.e., used different ages to stratify earlier diagnosis) to contribute to precise risk estimates by age of the relative at CRC diagnosis. ${ }^{2,24}$

In the Swedish Family Cancer database, data from 7 million people representing more than 2 million families were linked with the Swedish Cancer registry. ${ }^{27}$ The individuals represented offspring born after 1934 who had at least two siblings and their parents. Having a parent with CRC was associated with a doubling of risk; having a parent diagnosed younger than age 60 was associated with tripling of risk. ${ }^{27}$ One Chinese study looked at risk associated with affected siblings versus affected parents and did not suggest greater impact of sibling CRC over parental CRC. ${ }^{26}$

Three studies provided relative risks stratified by the age of the person at risk. In the Swedish database, the presence of a 
Table 2 Risk of CRC associated with family history of CRC

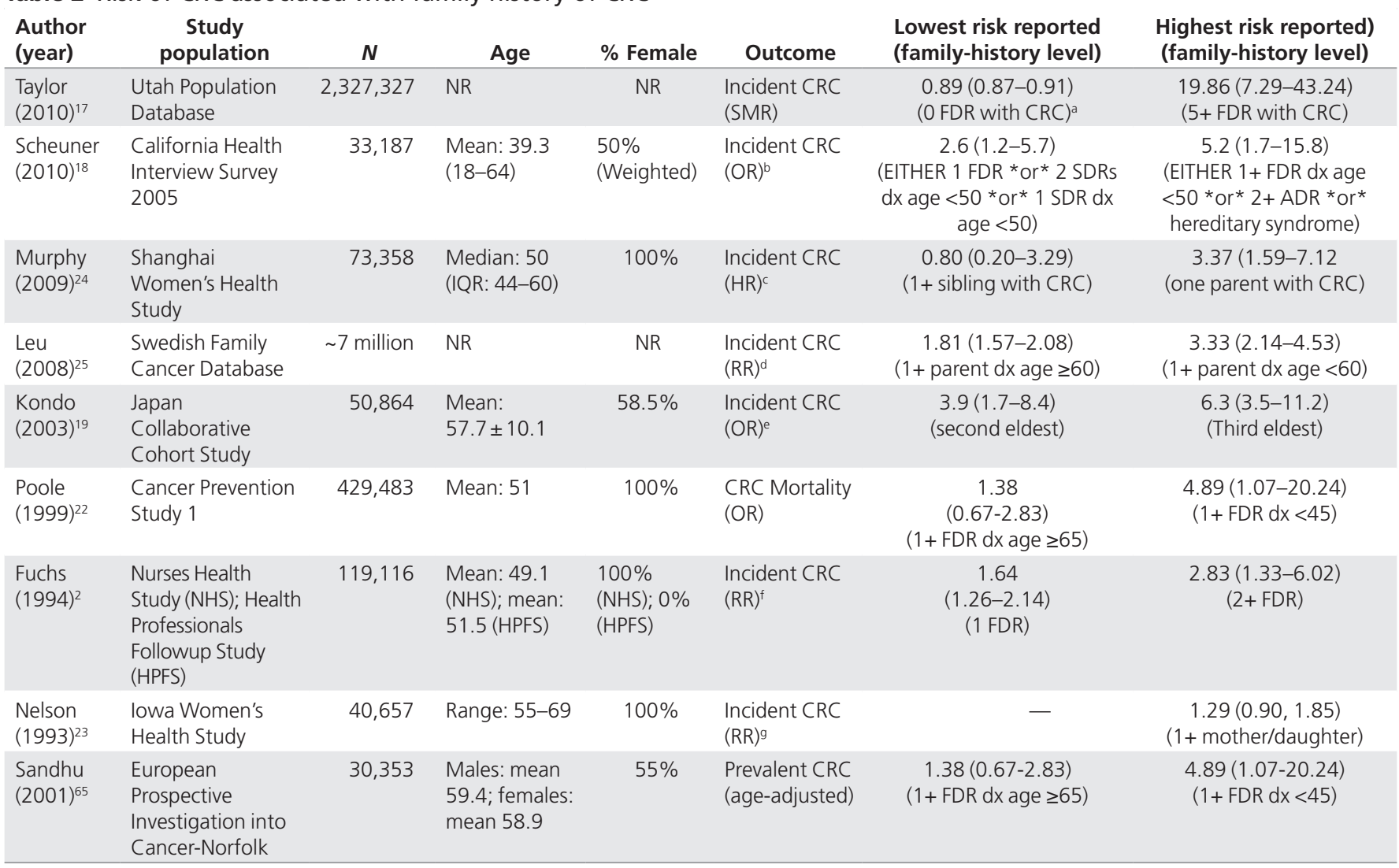

$A D R$, any-degree relative; CRC, colorectal cancer; dx, diagnosed; FDR, first-degree relative; HPFS, Health Professional Followup Study; HR, hazard ratio; IQR, interquartile range; NR, not reported; OR, odds ratio; RR, relative risk; SDR, second-degree relative; SMR, standardized morbidity ratio.

ased the population risk as comparison group; all other studies used "no family history" group as comparison. 'Adjusted for age, sex, race/ethnicity, education level, marital status, annual income, and personal history of cancer other than cancer of interest or nonmelanoma skin cancer. Women who reported no family history of cancer were used as referent group. Analyses adjusted for age, smoking, family income, education, BMI, physical activity, and diabetes. ${ }^{\mathrm{d} A d j u s t e d}$ for age. ${ }^{\mathrm{e}} \mathrm{Adjusted}$ for age at interview, number of brothers/sisters, race, education, smoking, BMI, age at menarche, number of pregnancies, and age at first pregnancy. ${ }^{\dagger} A d j u s t e d$ for age, screening endoscopy, smoking, alcohol consumption, aspirin use, BMI, physical activity, total energy intake, and energy adjusted nutrient.

parental history of CRC more than quadrupled CRC risk in adults aged $30-39$ years as compared with others the same age with no family history. ${ }^{27}$ Within each age decile of the person at risk, parental cancer history was associated with increased relative risks as compared with people without an affected parent of the same age. Relative risks remained elevated until at least age 70. Data from the Health Professionals Followup Study and Nurses' Health Study populations were generally consistent. ${ }^{2}$ British data regarding prevalent, rather than incident, cancer suggest decreasing risk with increasing age of the person at risk, but CIs were wide; also, this study population did not include people younger than 50 , in whom the risk may be most exaggerated. ${ }^{23}$ Overall, the preponderance of data suggests that history of one or more FDR with CRC is associated with a smaller and diminishing incremental increase in relative risk in those aged 50 years and older, as the prevalence of family history in the population increases. Thus, the relative impact of family history on preventable cancers in those younger than age $50-55$ years will be much greater than in older adults (see Supplementary Material online).
Critical appraisal concerns: risk and prevalence studies. All but one ${ }^{22}$ study controlled for age of the person at risk, and five controlled for the sex of the person at risk., ${ }^{2,1922,23,25}$ Only one study ${ }^{21}$ adjusted for family size, and no studies reported whether or how relatedness of the study participants was addressed. Only two studies used verified family histories, both with registry-based outcomes. ${ }^{19,27}$ All the others used self-report. Studies of non-US populations may have limited generalizability to US populations.

\section{Natural history}

Adenoma summary. There are very few data with which to make a strong conclusion about adenoma and family history. Data from two studies suggest a higher prevalence of adenomas in people with a positive family history of CRC as compared with people with no family history. There was a higher prevalence of two or more adenomas in people with a positive family history of CRC as compared with people with no family history. No evidence suggested differential adenoma location by family-history status. There is no 
evidence to suggest a differential prevalence of advanced adenoma in people with a family history of CRC.

Cancer summary. Very limited data were available regarding family history, natural history, and CRC. There was no evidence for difference in age at CRC diagnosis or stage at diagnosis by family-history status. There was mixed evidence regarding tumor location depending on family-history status. Some evidence suggested that those with a positive family history are more likely to have distal tumors, whereas others reported no difference.

We identified nine unique studies: three with relevant adenoma outcomes ${ }^{28-30}$ and six with relevant CRC outcomes. ${ }^{31-36}$
Adenoma study details. We identified three studies of a total of 35,590 people with family history-specific data on adenoma prevalence (two studies), advanced adenomas (three studies), multiple adenomas (three studies), or adenoma location (one study). ${ }^{28-30}$ In a study of 27,650 men enrolled in the Health Professionals Follow-up Study (HPFS) from 1986 to 2004, ${ }^{28}$ adenoma prevalence was increased in people with exactly one affected (1) FDR (15.4\%) or 2+ affected FDRs (19.1\%) as compared with people with no family history (10.0\%) (Table 3 ). Adenomas were more common in people with family history at all age groups, particularly at younger ages, based on slopes of weighted regression lines (raw data not provided). A German population-based cross-sectional study of colonoscopies of

Table 3 Natural history: adenoma

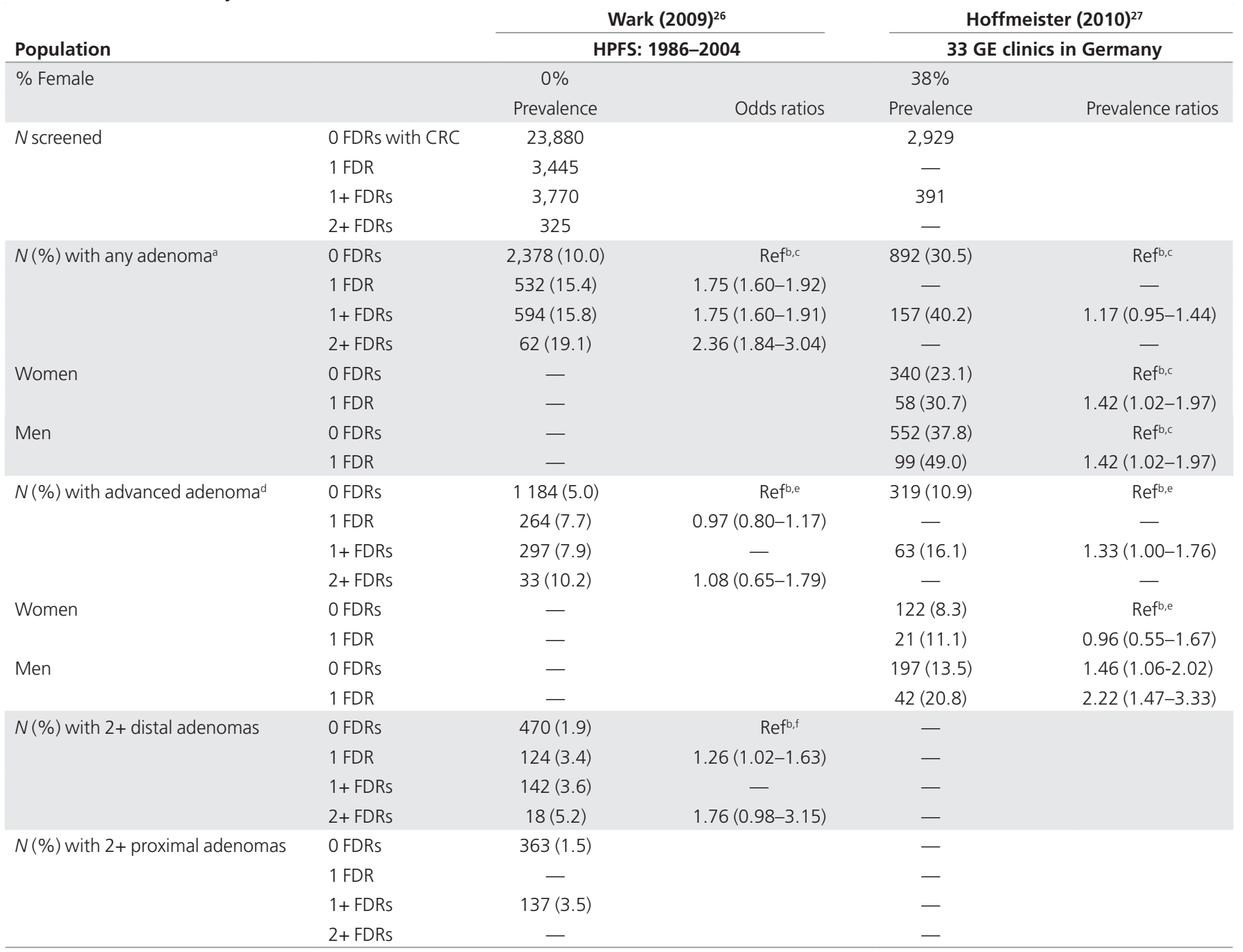

BMI, body mass index; CRC, colorectal cancer; FDR, first-degree relative; GE, gastroenterology; HPFS, Health Professional Followup Study; HRT, hormone replacement therapy; Ref, reference.

aWark: nonadvanced and advanced adenomas; Hoffmeister: nonadvanced and advanced adenomas and CRC. bWark: adjusted age, previous endoscopy, indication, aspirin, multivitamin, smoking, red meat, alcohol, folate, calcium, BMI, physical activity, total energy intake; Hoffmeister: age at colonoscopy, gender, education, HRT use, BMI, physical activity, alcohol, red meat consumption. 'Wark: odds ratio: adenoma versus no adenoma within each family-history level; Hoffmeister: prevalence ratio between family-history levels. ${ }^{\text {WWark: }}$ size $\geq 10 \mathrm{~mm}$ * or $^{*}$ tubulo/villous structure * or* carcinoma in situ; Hoffmeister: size $\geq 10 \mathrm{~mm}{ }^{*}$ or ${ }^{*}$ tubulo/villous structure * or* severe dysplasia *or* CRC. eWark: Odds ratio: advanced adenoma versus nonadvanced adenoma within each family-history level; Hoffmeister: prevalence ratio between family-history levels. "Wark: rates of multiple versus single adenoma. "-" is NR, not reported. 
3,320 people at average risk found a similar trend, with higher adenoma prevalence than the HPFS for those with no affected FDRs (30 vs. $10 \%$ ) and $1+$ FDR (40 vs. $15 \%) .{ }^{29}$

The HPFS suggested that the odds of advanced adenoma associated with family history were similar to those for any adenoma. ${ }^{28}$ In the German study, advanced adenoma was more prevalent in men than women regardless of family history (Table 3) ${ }^{29}$ The German study included CRC in an "advanced neoplasia" category ( $3.9 \%$ of total neoplasia; data not shown).

The HPFS analysis provided data on $2+$ adenomas by family history and was limited to distal location. ${ }^{28}$ It suggested an increasing prevalence of $2+$ adenomas with increasing family history. The adjusted odds of multiple adenomas remained when compared with either single adenomas or no adenomas.

A screening study of Veterans Affairs patients $(n=3,121$, 96.8\% male; age 50-75) did not describe the family history of the entire study population but reported a higher rate of family history (1+ parent or sibling) in people with two or more adenomas (19.3\%) as compared with people with one adenoma (12.9\%) or no polyps (12.2\%) (odds ratio (OR): 1.73; 95\% CI: 1.32-2.26); similarly, the study reported a higher rate of family history in people with advanced adenoma (18.4\% with advanced adenoma versus $15.8 \%$ with any adenoma (OR for advanced adenoma versus no polyp group: 1.62 , 95\% CI: 1.162.26; OR for any adenoma versus no polyp group: $1.36,95 \% \mathrm{CI}$ : $1.09-1.70) .{ }^{30}$ Only the German study reported adenoma location by family history (proximal/distal and colon/rectum); it found no significant difference in the distribution of location according to family history. ${ }^{28}$

Critical appraisal: adenoma studies. The study populations of two of the three studies were almost exclusively male, limiting their generalizability to women, ${ }^{28,30}$ but otherwise included populations of screening-relevant age. Three studies assessed natural history outcomes via study colonoscopy; ${ }^{29,30}$ one used self-report but verified positive findings with medical records. ${ }^{28}$ All studies included adequate follow-up time to detect the findings of interest, either by cross-sectional analysis of colonoscopy findings or by longitudinal design. Three studies relied on self-report of family history, ${ }^{28,30}$ one with verification of self-report via medical records or death certificates. ${ }^{29}$

Cancer studies. Four studies of 4,537 people reported data regarding age at diagnosis by family history of CRC. . $^{31,32,34,35}$ A study of 3,383 surgical CRC patients in Taiwan found no difference in age at detection in those with zero or one affected FDR but a younger age at detection for those with 2+ FDRs. However, this group included Lynch syndrome patients and therefore may reflect differential surveillance. An analysis of 1,001 women enrolled in the Nurses' Health Study found no difference in age at diagnosis according to family history. ${ }^{34}$

Two studies reported age at diagnosis by family history. A randomized trial for adjuvant therapy for stage III colon cancer in people within 56 days of surgery for a primary tumor did not find any baseline difference in age at trial enrollment by family history ${ }^{35}$ A US registry-based analysis of incident cancer between 1994 and 1996 did not find any difference between age at diagnosis for colon cancer by family history but found younger age at diagnosis for rectal cancer in people with no family history. ${ }^{31}$

Four studies reported data by family history on location of CRC at diagnosis. Registry-based studies in Sweden and Japan suggested the majority of cancers in people with a family history were distally located. ${ }^{33,36}$ However, two other studies found a more even distribution of distally and proximally located cancers. $^{32,34}$ In three studies providing data regarding stage at diagnosis by family history, there was no evidence of differential distributions of stage in people with a family history of CRC as compared with those without. ${ }^{31-33}$

Critical appraisal concerns: cancer studies. The natural history of CRC is difficult to assess because of the known effectiveness of treatment for detected CRC. Age at diagnosis was obtained at study enrollment for two studies ${ }^{32,35}$ and from registry data for one study $;^{33,37}$ one study collected self-reported data but verified positive results with medical records ${ }^{34}$ Family history was assessed by interview, ${ }^{31,32}$ self-report, ${ }^{34,35}$ or from registry records. ${ }^{33,37}$ No studies reported measures of relatedness or family size.

\section{Adherence}

Summary. Individuals with a positive family history are 1.4-3.3 times more likely to be adherent to CRC screening recommendations than individuals with no family history. One study, which objectively measured both family history and screening behavior, suggested a $7-8 \%$ absolute increase in screening adherence in people with positive family history.

Study details. We assessed three studies published since 2009, plus eight studies from the systematic review used as primary evidence, ${ }^{15}$ for a total of 11 studies. Ten were cross-sectional studies, together representing nine independent samples and 129,942 people 50 years of age and older (Table 4). All but one study $^{38}$ used a relatively nonspecific family-history definition of $1+$ FDR with CRC..$^{38}$ Only the three newer studies included age at diagnosis of the affected relative (age younger than 50 years) as a separate analysis. ${ }^{38-40}$ Three analyzed colonoscopy adherence alone; ${ }^{39-41}$ all others considered adherence to recommended CRC screening modalities (colonoscopy, sigmoidoscopy, and/ or fecal occult blood test). Eight studies published adherence rates ${ }^{38-40,42-46}$ three published relative risks of adherence for those with family history as compared with those with no family history.

An analysis of the 2005 California Health Interview Survey included 10,310 adults aged $50-64,7.5 \%$ of whom had a selfreported family history of CRC; ${ }^{38}$ those with a family history of $1+$ FDR or $2+$ SDRs were more than twice as likely to be adherent to CRC screening recommendations (OR: 2.77; 95\% CI: 2.20-3.49). Absolute adherence was increased by more than 25\%. In an analysis of 2008 Oregon Behavioral Risk Factor 
Table 4 Screening adherence by family history

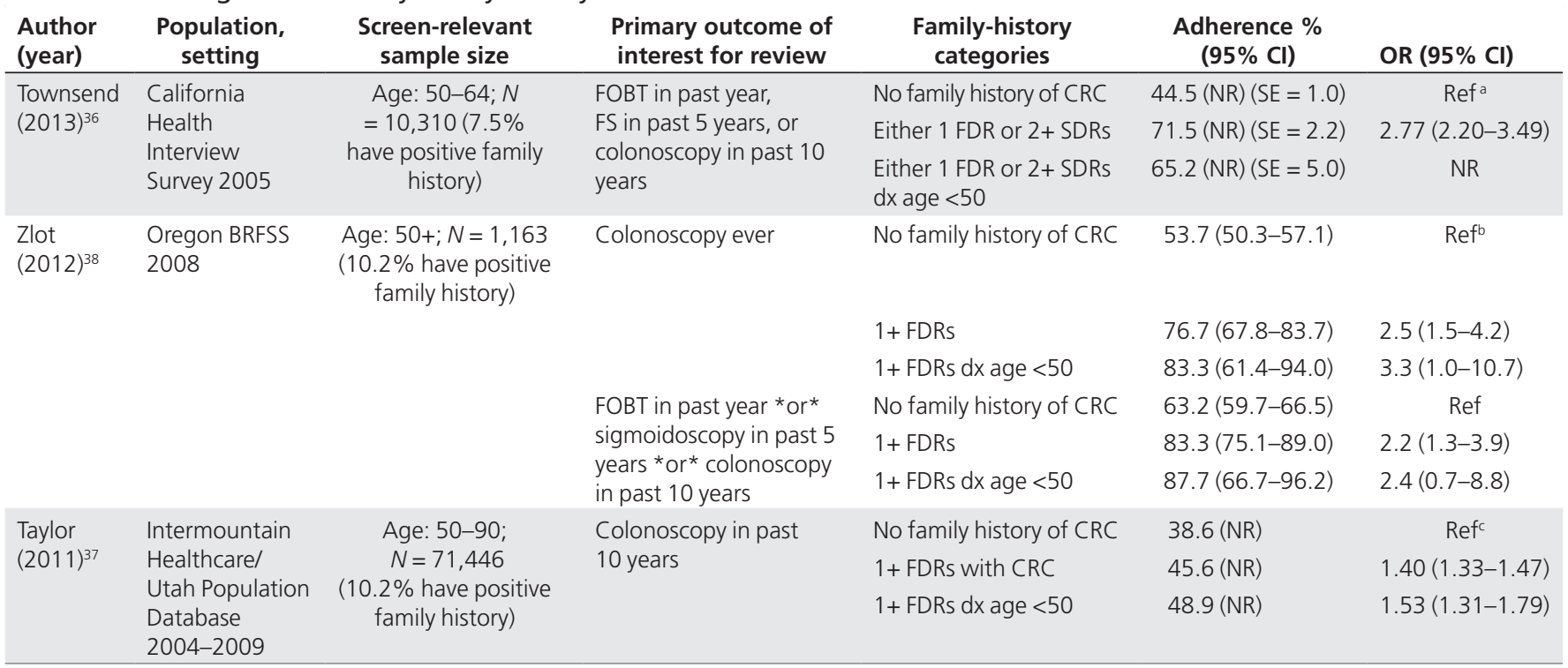

BRFSS, Behavioral Risk Factor Surveillance System; Cl, confidence interval; CRC, colorectal cancer; dx, diagnosed; FDR, first-degree relative; FS, flexible sigmoidoscopy; NR, not reported; Ref, reference; OR, odds ratio; SDR, second-degree relative.

adjusted for age, race/ethnicity, household income, health insurance, usual health-care provider, education. bAdjusted for education and smoking status. 'Adjusted for age, sex.

Surveillance System data, adherence was more than twice as likely for those with $1+$ FDR, regardless of screening criteria used, although estimates were less precise, probably due to sample size. ${ }^{40}$ Absolute adherence was at least $20 \%$ greater in those with a self-reported family history and was somewhat higher when not requiring colonoscopy.

In an analysis of the Utah Population Database 2004-2009, ${ }^{39}$ cancer history was confirmed via the Utah Cancer Registry and family relationships were established using comprehensive statewide genealogy data. Adherence to colonoscopy in the previous 10 years was increased in those with $1+\mathrm{FDR}$, but with somewhat attenuated relative (OR: 1.40; 95\% CI: 1.33-1.47) and absolute effects (7\% higher adherence) as compared with those of the two other studies. This study is the only one to use systematic, objective approaches to assess family history and colonoscopy rather than self-reported data, which could explain the more modest effect size.

The eight older studies consistently found an approximate doubling of the odds of reporting compliance with recommended CRC screening, regardless of the definitions of recommended CRC screening or population, corresponding to adherence rates of $26-50 \%$ for people with no family history as compared with $44-68 \%$ for people with $1+$ FDR. Precision varied with sample size, and data to evaluate screening adherence with colonoscopy versus other modalities were limited and mixed (Supplementary Material online).

Critical appraisal concerns: adherence studies. Ten of 11 studies gathered both exposure and outcome data via selfreport, and only one used registries and medical records to verify family history and screening use. ${ }^{39}$ Survey-based studies found varying response rates, from a quite low response rate of
$29 \%{ }^{38}$ to a high rate of $79 \% .{ }^{44}$ Four studies did not report the response rate, so response bias could not be assessed. . $^{40,45,47,48}$

\section{DISCUSSION}

We conducted a systematic review to identify the most current, highest-quality evidence of the prevalence of family history of colorectal cancer in the US population, as well as its influence on CRC risk, natural history, and screening adherence. These data provide important insights to clinicians and researchers, as well as supporting further modeling of effective and efficient evidence-based screening recommendations for those at more precisely increased risk of CRC due to more completely defined family histories. Our study suggests that objective measures of prevalence of family history may be lower than commonly cited and that the increased risk associated with family history is significant and may be associated with adenoma number rather than with faster adenoma progression. People with family history were more likely to adhere to screening than those without. Taken together, these data reinforce the need for optimal screening strategies for people with family history of CRC.

Prevalence. Our findings suggest that the prevalence of family history may be lower than the commonly discussed estimate of $10 \%$, which is likely based on self-reported data. This may reflect our emphasis on studies that focused on population samples rather than screening studies to minimize bias due to selective volunteering of patients and families with positive family histories. Although the prevalence of SDRs was higher than that of FDRs, ${ }^{19}$ family history considering various family patterns involving second- or third-degree relatives was never associated with more than a doubling of CRC risk. This suggests 
that for clinical purposes, determining the history of cancer in all FDRs may be both sufficient and the most feasible. Younger age at FDR diagnosis with CRC was relatively consistently associated with further increased risk, but current data made the degree of additional risk difficult to quantify. Only one study reported the prevalence of family history by race but suggested a prevalence at odds with actual increased CRC prevalence in African Americans. ${ }^{20}$ If these data are correct, then family history may be under-reported, less well known, or not a primary influence on the excess CRC burden in African Americans. One study suggested a higher prevalence of family history in females, suggesting gender differences in reporting consistent with other studies. ${ }^{18,49}$

Risk. Our review of population-based risk estimates suggested a range of risk from 0.89 (for individuals with no family history) to nearly a 20 -fold risk for individuals with likely inherited syndromes, with risk levels in between depending on the family-history configuration. This is consistent with those in other reviews ${ }^{50,51}$ suggesting increasing risk of CRC with increasing burden of family history. Our review has the added benefit of using only population-based study populations and adds results from a high-quality study using a comprehensive US-based population. ${ }^{19}$ This study used the entire population as the comparator in risk estimates, allowing subpopulation estimates that ranged from a modestly reduced risk to an exaggerated risk for the very few with five or more FDRs. Importantly, relative risks for CRC varied between 1.9 and 4.4 in those with at least one FDR, depending on the number of relatives and age at diagnosis. It also included relative risk estimates stratified by age of the person at risk. This study represents a population with potentially lower behavioral risk factors, such as smoking and alcohol use, than the general population. Concern is frequently raised about how genetically representative this population is, but the UPDB is genetically representative of US Caucasian and northern European populations with a low level of inbreeding. ${ }^{52-54}$ This study is of high quality and its methods should be replicated whenever possible using other data sources.

Evidence for adenoma and/or CRC risk in people with a family history of adenoma (rather than CRC) is limited; no studies of this type met our best-evidence criteria. One review found only two studies, both with methodologic flaws. ${ }^{55}$ An article published after our search ended suggests increased risk of both CRC and adenoma in people with family history of adenoma. ${ }^{56}$

Natural history. Our best evidence suggests a higher prevalence of adenomas in people with family history of CRC in men, consistent with previous work, ${ }^{57}$ but does not suggest that adenoma progression (measured by advanced adenoma) is accelerated in people with family history. Small studies have suggested that adenoma growth may be accelerated in people with a family history of CRC. ${ }^{58,59}$ Future, larger studies could provide additional insight into the impact of family history on adenoma progression. Evolving temporal trends in adenoma classification, such as the sessile serrated pathway, made it challenging to pool data from multiple studies.

Two studies suggested a trend toward increased multiple adenomas in people with a family history of CRC. Our best evidence did not suggest an association between adenoma location and family history. For colorectal cancer, the data were even more limited but suggested no difference in the age or stage at diagnosis by family-history status. There was very limited, conflicting evidence regarding tumor location and family history. Very limited data suggest that CRC progression in people with family history of CRC is similar to that in the general population.

Adherence. Our review confirms the findings of a previous review suggesting a clear association between family history of CRC and adherence to both colonoscopy and other CRC screening modalities. ${ }^{15}$ Increased adherence likely reflects both organizational (physician recommendation, access) and patient-level factors such as risk perception, but it is worth noting that adherence remains low in all groups. Only one study provided data using objectively measured family history and screening; these data may provide the best estimates for adherence for those with a positive family history but are limited to a single geographic location with a somewhat different social environment.

Our best-evidence approach allowed us to summarize an extensive, heterogeneous body of research but may have resulted in our excluding some relevant data. To our knowledge, this is the first review to limit estimates of prevalence of family history and CRC risk to population-based studies, which may be the least biased for informing modeling of populationbased screening.

We were also limited by the heterogeneous nature of familyhistory reporting in the literature. There is often an analytical rationale for collapsing family-history strata to increase sample size, but this makes it difficult to pool data across studies. We excluded 41 studies based on the lack of a basic definition that included number and degree of relatives affected, and a further 43 for not providing data stratified by family history. In the remaining studies, more than half used our minimum familyhistory definition of "at least one FDR." Few studies reported age at diagnosis of the affected relative, verified the accuracy of self-reported data or used objective measures, or assessed or adjusted for family size.

We recommend that future research define family history as, at minimum, the number and degree of affected relatives, and that raw data be reported to maximize potential for aggregating study data. Family-history studies should also report how history was assessed, whether and how it was verified, and family size. Measures of how often family history changes in ways that materially affect an individual's CRC risk as age increases would help reduce the uncertainty associated with family history at younger ages and the frequency with which family history for CRC should be updated. High-quality studies of family 
history and CRC in populations of non-European ancestry are also needed.

Family history is an imperfect and dynamic measure. As family sizes decrease over time, fewer relatives are available to define risk. Also, as endoscopic screening with curative intervention increases, family history can be hidden in families if affected persons are more likely to communicate a diagnosis of advanced cancer to their relatives than a polyp removed during endoscopy. However, family history of CRC remains a clinically meaningful way to identify individuals at increased risk for $\mathrm{CRC}$, and it may be the most feasible approach at present, given that multifactorial risk assessment tools are not yet validated for clinical practice ${ }^{60-65}$ and the utility of genetic risk stratification is still being investigated. ${ }^{66-68}$ In the future, risk based on polygenic and/or multifactorial risk assessment may augment family history-based approaches. ${ }^{69}$ Family-history collection and reporting should continue in ways that are conducive to knowledge development.

\section{SUPPLEMENTARY MATERIAL}

Supplementary material is linked to the online version of the paper at http://www.nature.com/gim.

\section{ACKNOWLEDGMENTS}

This study was supported by Centers for Disease Control and Prevention grant 5U18GD000076-02.

\section{DISCLOSURE}

The authors declare no conflict of interest.

\section{REFERENCES}

1. Howlader N, Noone AM, Krapcho M, et al. SEER Cancer Statistics Review, 1975-2009 (Vintage 2009 Populations). http://seer.cancer.gov/csr/1975_2009_ pops09/. Accessed 7 January 2014.

2. Fuchs CS, Giovannucci EL, Colditz GA, Hunter DJ, Speizer FE, Willett WC. A prospective study of family history and the risk of colorectal cancer. N Eng/ J Med 1994:331:1669-1674.

3. Smith RA, von Eschenbach AC, Wender R, et al.; ACS Prostate Cancer Advisory Committee, ACS Colorectal Cancer Advisory Committee, ACS Endometrial Cancer Advisory Committee. American Cancer Society guidelines for the early detection of cancer: update of early detection guidelines for prostate, colorectal, and endometrial cancers. Also: update 2001-testing for early lung cancer detection. CA Cancer J Clin 2001;51:38-75; quiz 77.

4. Levin $B$, Rozen P, Young $G$. How should we follow up colorectal premalignant conditions? In: Rozen P, Young G, Levin B, Spann SJ (eds). Colorectal Cancer in Clinical Practice: Prevention, Early Detection, and Management. Martin Dunitz: London, UK, 2002:67-76.

5. Centers for Disease Control and Prevention. Vital signs: colorectal cancer screening test use_-United States, 2012. MMWR 2013;62:881-888.

6. Joseph DA, DeGroff AS, Hayes NS, Wong FL, Plescia M. The Colorectal Cancer Control Program: partnering to increase population level screening. Gastrointest Endosc 2011;73:429-434.

7. Levin B, Lieberman DA, McFarland B, et al. Screening and surveillance for the early detection of colorectal cancer and adenomatous polyps, 2008: a joint guideline from the American Cancer Society, the US Multi-Society Task Force on Colorectal Cancer, and the American College of Radiology. Gastroenterology 2008;134:1570-1595.

8. American Cancer Society. Colorectal Cancer, 2014. http://www.cancer.org/ cancer/colonandrectumcancer/index. Accessed 7 January 2014.

9. American Gastroenterological Association. American Gastroenterological Association Home Page, 2014. http://www.gastro.org/. Accessed 7 January 2014.
10. Canadian Task Force on Preventive Health Care. Guidelines, 2014. http:// canadiantaskforce.ca/guidelines/. Accessed 7 January 2014.

11. US Preventive Services Task Force. Screening for Colorectal Cancer: U.S. Preventive Services Task Force Recommendation Statement. AHRQ Publication 08-05124-EF-3. 2008. http://www. uspreventiveservicestaskforce.org/uspstf08/ colocancer/colors.htm. Accessed 7 January 2014.

12. Rex DK, Johnson DA, Anderson JC, Schoenfeld PS, Burke CA, Inadomi JM: American College of Gastroenterology. American College of Gastroenterology guidelines for colorectal cancer screening 2009 [corrected]. Am J Gastroenterol 2009;104:739-750

13. Cancer Intervention and Surveillance Modeling Network. Colorectal Cancer Model Profiles. 2014. http://cisnet.cancer.gov/colorectal/profiles.html. Accessed 7 January 2014.

14. Treadwell JR, Singh S, Talati R, McPheeters ML, Reston JT. A framework for best evidence approaches can improve the transparency of systematic reviews. J Clin Epidemiol 2012;65:1159-1162.

15. Holden DJ, Harris R, Porterfield DS, et al. Enhancing the use and quality of colorectal cancer screening. Evidence Report/Technology Assessment 2010;2010:1-195.

16. Whitlock EP, Lin JS, Chou R, Shekelle P, Robinson KA. Using existing systematic reviews in complex systematic reviews. Ann Intern Med 2008;148:776-782.

17. Kleihues P, Sobin LH. World Health Organization classification of tumors. Cancer 2000:88:2887.

18. Qureshi N, Carroll JC, Wilson B, et al. The current state of cancer family history collection tools in primary care: a systematic review. Genet Med 2009; 11:495-506.

19. Taylor DP, Burt RW, Williams MS, Haug PJ, Cannon-Albright LA. Populationbased family history-specific risks for colorectal cancer: a constellation approach. Gastroenterology 2010;138:877-885.

20. Scheuner MT, McNeel TS, Freedman AN; The 2005 California Health Interview Survey. Population prevalence of familial cancer and common hereditary cancer syndromes. Genet Med 2010;12:726-735.

21. Kondo $T$, Toyoshima $H$, Tsuzuki $Y$, et al. Aggregation of stomach cancer history in parents and offspring in comparison with other sites. Int J Epidemiol 2003:32:579-583.

22. Pinsky PF, Kramer BS, Reding D, Buys S; PLCO Project Team. Reported family history of cancer in the prostate, lung, colorectal, and ovarian cancer screening trial. Am J Epidemiol 2003;157:792-799.

23. Sandhu MS, Luben R, Khaw KT. Prevalence and family history of colorectal cancer: implications for screening. J Med Screen 2001;8:69-72.

24. Poole CA, Byers T, Calle EE, Bondy J, Fain P, Rodriguez C. Influence of a family history of cancer within and across multiple sites on patterns of cancer mortality risk for women. Am J Epidemiol 1999;149:454-462.

25. Nelson CL, Sellers TA, Rich SS, Potter JD, McGovern PG, Kushi LH. Familial clustering of colon, breast, uterine, and ovarian cancers as assessed by family history. Genet Epidemiol 1993;10:235-244.

26. Murphy G, Shu XO, Gao YT, et al. Family cancer history affecting risk of colorectal cancer in a prospective cohort of Chinese women. Cancer Causes Control 2009;20:1517-1521.

27. Leu M, Reilly M, Czene K. Evaluation of bias in familial risk estimates: a study of common cancers using Swedish population-based registers. J Nat/ Cancer Inst 2008;100:1318-1325.

28. Wark PA, Wu K, van 't Veer P, Fuchs CF, Giovannucci EL. Family history of colorectal cancer: a determinant of advanced adenoma stage or adenoma multiplicity? Int J Cancer 2009;125:413-420.

29. Hoffmeister M, Schmitz S, Karmrodt E, et al. Male sex and smoking have a larger impact on the prevalence of colorectal neoplasia than family history of colorectal cancer. Clin Gastroenterol Hepatol 2010;8:870-876.

30. Lynch KL, Ahnen DJ, Byers T, Weiss DG, Lieberman DA. First-degree relatives of patients with advanced colorectal adenomas have an increased prevalence of colorectal cancer. Clin Gastroenterol Hepatol 2003;1:96-102.

31. Zell JA, Honda J, Ziogas A, Anton-Culver H. Survival after colorectal cancer diagnosis is associated with colorectal cancer family history. Cancer Epidemiol Biomarkers Prev 2008;17:3134-3140.

32. Kao PS, Lin JK, Wang HS, et al. The impact of family history on the outcome of patients with colorectal cancer in a veterans' hospital. Int J Colorectal Dis 2009;24:1249-1254.

33. Japanese Research Society; Registry Committee, Japanese Research Society for Cancer of the Colon and Rectum. Clinical and pathological analyses of patients with a family history of colorectal cancer. Jpn J Clin Oncol 1993;23:342-349.

34. Bass AJ, Meyerhardt JA, Chan JA, Giovannucci EL, Fuchs CS. Family history and survival after colorectal cancer diagnosis. Cancer 2008;112:1222-1229. 
35. Chan JA, Meyerhardt JA, Niedzwiecki D, et al. Association of family history with cancer recurrence and survival among patients with stage III colon cancer. JAMA 2008;299:2515-2523.

36. Hemminki K, Santi I, Weires M, Thomsen H, Sundquist J, Bermejo JL. Tumor location and patient characteristics of colon and rectal adenocarcinomas in relation to survival and TNM classes. BMC Cancer 2010;10:688.

37. Hemminki K, Granström C, Chen B. The Swedish family-cancer database: update, application to colorectal cancer and clinical relevance. Hered Cancer Clin Pract 2005;3:7-18.

38. Townsend JS, Steele CB, Richardson LC, Stewart SL. Health behaviors and cancer screening among Californians with a family history of cancer. Genet Med 2013;15:212-221.

39. Taylor DP, Cannon-Albright LA, Sweeney C, et al. Comparison of compliance for colorectal cancer screening and surveillance by colonoscopy based on risk. Genet Med 2011;13:737-743.

40. Zlot Al, Silvey K, Newell N, Coates RJ, Leman R. Family history of colorectal cancer: clinicians' preventive recommendations and patient behavior. Prev Chronic Dis 2012;9:E21.

41. Young WF, McGloin J, Zittleman L, West DR, Westfall JM. Predictors of colorectal screening in rural Colorado: testing to prevent colon cancer in the High Plains Research Network. J Rural Health 2007;23:238-245.

42. Peterson NB, Murff HJ, Ness RM, Dittus RS. Colorectal cancer screening among men and women in the United States. J Womens Health (Larchmt) 2007:16:57-65.

43. Seeff LC, Nadel MR, Klabunde CN, et al. Patterns and predictors of colorectal cancer test use in the adult U.S. population. Cancer 2004;100:2093-2103.

44. Shapiro JA, Seeff LC, Thompson TD, Nadel MR, Klabunde CN, Vernon SW. Colorectal cancer test use from the 2005 National Health Interview Survey. Cancer Epidemiol Biomarkers Prev 2008;17:1623-1630.

45. Schumacher MC, Slattery ML, Lanier AP, et al. Prevalence and predictors of cancer screening among American Indian and Alaska native people: the EARTH study. Cancer Causes Control 2008;19:725-737.

46. Zapka JG, Puleo E, Vickers-Lahti M, Luckmann R. Healthcare system factors and colorectal cancer screening. Am J Prev Med 2002;23:28-35.

47. Wong ST, Gildengorin G, Nguyen T, Mock J. Disparities in colorectal cancer screening rates among Asian Americans and non-Latino whites. Cancer 2005;104(12 suppl):2940-2947.

48. Lemon S, Zapka J, Puleo E, Luckmann R, Chasan-Taber L. Colorectal cancer screening participation: comparisons with mammography and prostate-specific antigen screening. Am J Public Health 2001;91:1264-1272.

49. Zallen D. To Test or Not to Test: A Guide to Genetic Screening and Risk. Rutgers University Press: New Brunswick, NJ, 2008.

50. Butterworth AS, Higgins JP, Pharoah P. Relative and absolute risk of colorectal cancer for individuals with a family history: a meta-analysis. Eur J Cancer 2006;42:216-227.

51. Johns LE, Houlston RS. A systematic review and meta-analysis of familial colorectal cancer risk. Am J Gastroenterol 2001;96:2992-3003.
52. Cannon-Albright LA, Thomas A, Goldgar DE, et al. Familiality of cancer in Utah. Cancer Res 1994;54:2378-2385.

53. Jorde LB. Inbreeding in the Utah Mormons: an evaluation of estimates based on pedigrees, isonymy, and migration matrices. Ann Hum Genet 1989;53(Pt 4):339-355.

54. McLellan T, Jorde LB, Skolnick MH. Genetic distances between the Utah Mormons and related populations. Am J Hum Genet 1984;36:836-857.

55. Imperiale TF, Ransohoff DF. Risk for colorectal cancer in persons with a family history of adenomatous polyps: a systematic review. Ann Intern Med 2012;156:703-709.

56. Tuohy TM, Rowe KG, Mineau GP, Pimentel R, Burt RW, Samadder NJ. Risk of colorectal cancer and adenomas in the families of patients with adenomas: a population-based study in Utah. Cancer 2014;120:35-42.

57. Wilschut JA, Habbema JD, Ramsey SD, Boer R, Looman CW, van Ballegooijen M. Increased risk of adenomas in individuals with a family history of colorectal cancer: results of a meta-analysis. Cancer Causes Control 2010;21:2287-2293.

58. Almendingen $\mathrm{K}$, Hofstad $\mathrm{B}$, Vatn $\mathrm{MH}$. Does a family history of cancer increase the risk of occurrence, growth, and recurrence of colorectal adenomas? Gut 2003;52:747-751.

59. Fossi $S$, Bazzoli F, Ricciardiello $L$, et al. Incidence and recurrence rates of colorectal adenomas in first-degree asymptomatic relatives of patients with colon cancer. Am J Gastroenterol 2001;96:1601-1604.

60. National Cancer Institute. Colorectal Cancer Risk Assessment Tool. 2010. http:// www.cancer.gov/colorectalcancerrisk/. Accessed 10 December 2013.

61. Schroy PC 3rd, Coe AM, Mylvaganam SR, et al. The Your Disease Risk Index for colorectal cancer is an inaccurate risk stratification tool for advanced colorectal neoplasia at screening colonoscopy. Cancer Prev Res (Phila) 2012;5:1044-1052.

62. ClinRisk. Welcome to QCancer. 2013. http://www.qcancer.org/. Accessed 10 December 2013

63. Yeoh KG, Ho KY, Chiu HM, et al.; Asia-Pacific Working Group on Colorectal Cancer. The Asia-Pacific Colorectal Screening score: a validated tool that stratifies risk for colorectal advanced neoplasia in asymptomatic Asian subjects. Gut 2011;60:1236-1241.

64. Wei EK, Giovannucci E, Wu K, et al. Comparison of risk factors for colon and rectal cancer. Int J Cancer 2004;108:433-442.

65. Wei EK, Colditz GA, Giovannucci EL, Fuchs CS, Rosner BA. Cumulative risk of colon cancer up to age 70 years by risk factor status using data from the Nurses' Health Study. Am J Epidemiol 2009;170:863-872.

66. Jiao $S$, Peters $U$, Berndt $S$, et al. Estimating the heritability of colorectal cancer. Human Mol Genet 2014;23:3898-3905.

67. Dunlop MG, Tenesa A, Farrington SM, et al. Cumulative impact of common genetic variants and other risk factors on colorectal cancer risk in 42103 individuals. Gut 2013:62:871-881.

68. Khoury MJ, Janssens AC, Ransohoff DF. How can polygenic inheritance be used in population screening for common diseases? Genet Med 2013;15:437-443.

69. Ghosh A, Hartge P, Kraft P, et al. Leveraging family history in population-based case-control association studies. Genet Epidemio/ 2014;38:114-122. 